\title{
Part-of-Speech Classification from Magnetoencephalography Data Using 1-Dimensional Convolutional Neural Network
}

\author{
Alessandro Lopopolo \\ Center for Language Studies \\ Radboud University \\ Nijmegen, the Netherlands. \\ a.lopopolo@let.ru.nl
}

\author{
Antal van den Bosch \\ Meertens Institute - KNAW \\ Amsterdam, the Netherlands. \\ Center for Language Studies \\ Radboud University \\ Nijmegen, the Netherlands. \\ a.vandenbosch@let.ru.nl
}

\begin{abstract}
Neural decoding of speech and language refers to the extraction of information regarding the stimulus and the mental state of subjects from recordings of their brain activity while performing linguistic tasks. Recent years have seen significant progress in the decoding of speech from cortical activity. This study instead focuses on decoding linguistic information. We present a deep parallel temporal convolutional neural network (1DCNN) trained on part-of-speech $(\mathrm{PoS})$ classification from magnetoencephalography (MEG) data collected during natural language reading. The network is trained on data from 15 human subjects separately, and yields above-chance accuracies on test data for all of them. The level of PoS was targeted because it offers a clean linguistic benchmark level that represents syntactic information, and abstracts away from semantic or conceptual representations. Our contribution provides a next step towards the direct decoding of linguistic information from brain activity, with potential implications for brain-computer-interface (BCI) and possibly neural prosthetics for communication.
\end{abstract}

\section{Introduction}

Neural decoding refers to the extraction of information about the stimuli or mental states from brain activity of human subjects. It generally consists of the application of machine learning techniques to electroencephalograpy (EEG), magnetoecephalograpy (MEG), functional magnetic resonance (fMRI), electrocorticography (ECoG), or other types of brain recordings. Here we report on exploratory work aimed at assigning the parts-ofspeech (PoS) tags of words to MEG data recorded during natural language reading. We present the results of a subject-level decoding experiment using 1-dimensional convolutional neural networks (1DCNN).

Recent years have seen a growth of interest in speech signal reconstruction from patterns of brain activity (Akbari et al., 2019). Advances in this type of research may contribute towards the development of brain computer interfaces (BCI) for communication, especially for patients unable to talk or communicate in any other ways. By describing the development of a 1DCNN classifier aimed at decoding linguistic information - and not speech - from brain recordings, this paper offers a different perspective. We aim to contribute to natural language decoding from brain activity by introducing an end-to-end system that returns PoStags directly from MEG recordings, without relying on other linguistic information. This proof-ofconcept study may offer a starting point for alternative BCI interfaces, e.g. for writing assistance.

Figure 1 displays the general structure of the classifier and the source of brain activity considered in the present experiment. We used MEG data from 13 left-hemisphere brain regions that are consistently reported in the literature as being involved in language processing and reading. These regions include the left Inferior Frontal Gyrus, the left Temporal Lobe, the left Angular and Supramarginal Gyrus and the Occipital Cortex. We focus on six high-frequency PoS-tags including nouns (henceforth $\mathrm{N}$ ), adjectives (ADJ), verbs (V), 
determiners (DET), pronouns (PRON), and prepositions (VS). We choose to focus on PoS tags because they offer a clean linguistic benchmark test that focuses on syntactic information, and abstracts away from semantic or conceptual representations.

\section{Related work}

The application of machine learning to EEG, MEG, fMRI, EcOG and other types of brain activity data has been extensively employed as a tool for studying the organization of conceptual, semantic and linguistic information in the brain. Among the most influential works in this direction are Haxby et al. (2001), Mitchell et al. (2008), and more recently Huth et al. (2016). Recent years have seen a multiplication of studies aimed at explaining brain activity using deep learning architectures. Most of these studies focused on finding a mapping between brain areas and layers or components of networks. One example is the linear mapping between regions of the visual cortex (occipital and ventro-temporal cortices) and image classification deep convolutional neural networks (Kuzovkin et al., 2018; Laskar et al., 2018).

Although we do not doubt the methodological and theoretical interest of such works, our focus, for the present experiment, is not on explaining the brain mechanisms underlying language processing, but on assessing the feasibility of applying a CNN architecture directly to the classification of MEG data.

We do take inspiration from pipelines developed in speech reconstruction and motor imagery classification, and we apply them to the classification of linguistic categories - PoS in our case from MEG data. Works such as those of Thomas et al. (2017), Tang et al. (2017), and Schirrmeister et al. (2017) applied deep learning on brain activity classification (mostly concerning motor imagery) for brain-computer interface (BCI), and reported significant improvements in accuracy as compared to systems that do not adopt deep learning techniques. In recent years there has been a flourishing of works aimed at directly reconstructing speech signals from brain activity (Herff et al., 2015; Akbari et al., 2019). In particular, Akbari et al. (2019) shows the great potential offered by deep learning techniques to reconstruct speech signal from intracranial encephalography (iEEG).

\section{MEG dataset}

We used the data of 15 randomly selected subjects belonging to the MEG section of the MOUS dataset (Schoffelen et al., 2019). All subjects were Dutch native speakers, who were asked to read (not aloud) 120 Dutch sentences presented on a screen word by word, containing a total of 1377 words. All sentences varied between 9 and 15 words in length. For more details on the subject pool, stimuli, data acquisition and preprocessing, we refer to the data paper publication paper (Schoffelen et al., 2019).

\subsection{Part-of-Speech tags}

The stimulus sentences contained words tagged with 9 different PoS tags. These tags consist of: noun $(\mathrm{N})$, determiner $(\mathrm{DET})$, verb $(\mathrm{V})$, adjective (ADJ), preposition (PREP), pronoun (PRON), adverb (ADV), conjunction (CONJ), and numeral (NUM). The tagset closely follows the practices of the Dutch Grammar 'Algemene Nederlandse Spraakkunst' (ANS) (Haeseryn et al., 1997) and consists of a subset of the base PoS tags used in the Spoken Dutch Corpus (CGN) (Oostdijk, 2000).

As Figure 2 shows, the frequency of these tags in the considered subset varies significantly with up to $22.74 \%$ for nouns (N) to down to $0.25 \%$ for numerals (NUM). We focus our analysis only on the top six most frequent tags: nouns, determiners, verbs, adjectives, prepositions and pronouns. Trials relative to words tagged with the lowfrequency PoS-tags adverbs, conjunctions, and numerals, are discarded from the analysis.

\subsection{Stimulation paradigm}

The sentence were presented visually with a LCD projector, with a vertical refresh rate of $60 \mathrm{~Hz}$ situated outside the MEG scanning rooms, and projected via mirrors onto the screen inside the measurement room. All stimuli were presented in a black mono-spaced font on a gray background at the center of the screen within a visual angle of 4 degrees. Sentences were presented word-by-word with a mean duration of $351 \mathrm{~ms}$ for each word (minimum of $300 \mathrm{~ms}$ and maximum of $1400 \mathrm{~ms}$, depending on word length). The duration of the stimuli was determined taking into account both the word length, the number of words in the whole sentence, and the number of letters within each word. Each word was separated by an empty screen for $300 \mathrm{~ms}$ before the onset of the next 




Figure 1: Schematic overview of the experiment. Activity from 13 distinct brain regions (listed on the top right, as Brodmann's Areas and their corresponding anatomical labels) is fed to a series of parallel 1D convolutional modules that converge to a series of stacked linear layers connected to an output layer predicting the PoS tag (bottom-left) of distinct trials of brain activity.

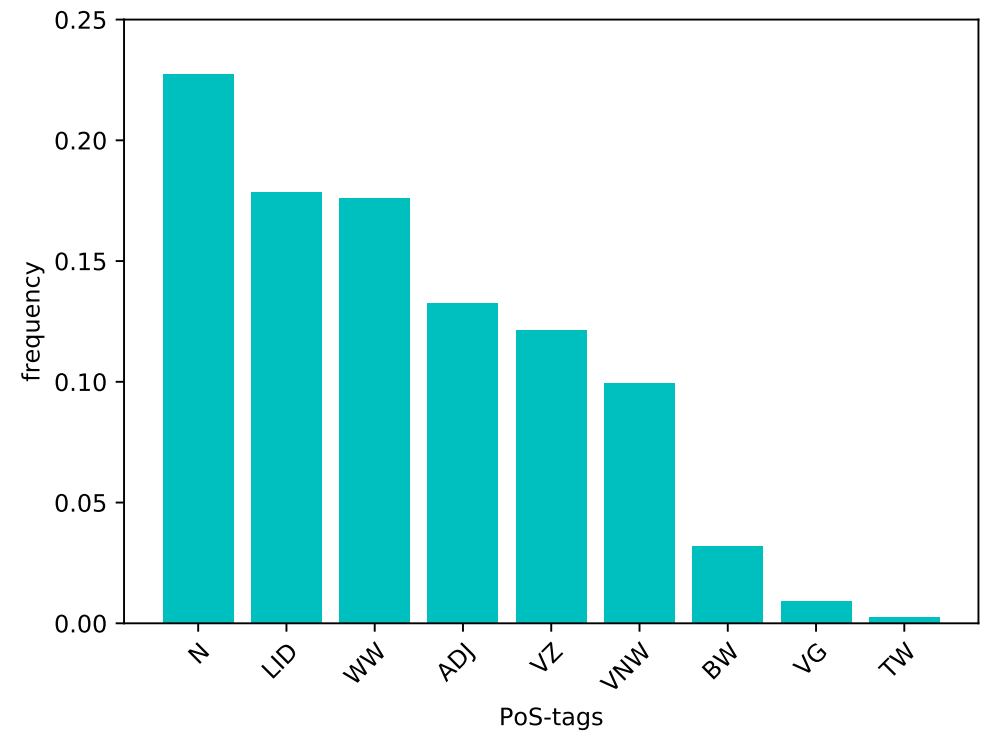

Figure 2: Frequency of each PoS-tag relative to the 15 subject stimulus sentences.

word.

\subsection{MEG data acquisition and pre-processing}

MEG data were collected with a 275-channel axial gradiometer system (CTF). The signals were digitized at a sampling frequency of $1200 \mathrm{~Hz}$ (cutoff frequency of the analog anti-aliasing low pass filter was $300 \mathrm{~Hz}$ ). The data was then baseline corrected and divided in trials corresponding to each word in the stimulus sentences. Trials containing artifacts were removed. The data was then transformed from sensor space to anatomical space composed of 378 cortical parcels.

\subsection{Cortical regions selection}

In order to reduce the size of the data, activity from only 78 cortical parcels were retained for the present experiments. These 78 parcels are grouped into 13 left hemisphere region considered central to language processing (Friederici 
and Gierhan, 2012). Regions are defined following Brodmann's cytoarchitectonic cortical parcellation (Brodmann's Areas, BA's). The following anatomical labels cover the 13 BA defined areas included in the experiment: Inferior Frontal Gyrus (IFG: BA44, BA45 and BA47), Superior Temporal Gyrus (STG: BA22), Inferior Temporal Gyrus (ITG: BA20), Middle Temporal Gyrus (MTG: BA21), Fusiform Gyrus (FG: BA37), Angular Gyrus (AG: BA39), Supramarginal Gyrus (SMG: BA40), Temporal Pole (TP: 38), and finally the Primary (V1: BA17), Secondary (V2: BA18) and Associative Visual Cortices (V3/4/5: BA19).

The left IFG is considered a central hub of language processing and production and is generally referred as Broca's Area. Similarly, the STG - including Wernicke's Area - is consistently found being active during language processing and its lesion correlates with processing deficits (Friederici et al., 2003; Catani et al., 2005; Hagoort, 2013). The left MTG and ITG are hypothesized being the center of lexical and conceptual semantic encoding (Binder and Desai, 2011). FG contains the Visual Word Form Area considered to be central in written language reading (Cohen, 2002). The left AG and SMG and the left TP are involved in language processing and usually interpreted as integrative hubs playing a connective role between other regions (Brennan et al., 2016). Finally, the visual cortex is also included, considering the visual nature of the stimulation paradigm employed during the collection of the MEG dataset.

Each of the 13 Brodmann's Areas are composed of a set of smaller brain partitions following Conte's brain partition atlas (Van Essen et al., 2011). These amount to a total of 78 cortical parcels, or sources of brain activity, available to our 1DCNN.

\subsection{Data structure}

The data for each subject is divided in trials. A trial $T$ is a tuple $\left(w_{i}: M\right)$ consisting of the recording of brain activity $M$ during the presentation of a word $w_{i} . M$ is a $[p \times t]$ matrix where $p$ are the anatomical parcels in which the original MEG sensor signal was projected and $t$ corresponds to the data point of the activity time series. In this experiment $p=78$ and $t=165$, corresponding to $550 \mathrm{~ms}$ of MEG activity at $300 \mathrm{~Hz}$ sampling rate. Therefore, given sentence $w_{1}, w_{2}, \ldots w_{n}$, the MEG dataset contain $n$ trials $T$ containing a [78 $\times 165$ ] matrix coupled with a word $w_{i}$. Each word in the stimulus sentences was manually assigned its $\mathrm{PoS}$ tag according to the Spoken Dutch Corpus standard (Eynde, 2004).

\section{Part-of-Speech decoding}

The goal of the present experiment is to develop and train a $\mathrm{CNN}$-based classifier that assigns to each trial of MEG data the PoS tag corresponding to the word that was presented during the acquisition of that trial.

\subsection{Decoder architecture}

We decided to use 1DCNN as the main component of the decoder. The motivation for this choice is that we want to leverage the high temporal resolution of the MEG data while preserving the anatomical information provided by the data set. The 78 anatomical regions are treated as separate channels by the CNN, so that 1 convolutional kernel is trained separately for each channel. The 1DCNN are organized in convolutional modules composed by one 1DCNN layer followed by batch normalization and dropout operations (see Figure 4). These later components are introduced to reduce the decoder's tendency to overfit (Ioffe and Szegedy, 2015; Hinton et al., 2012). The convolutional modules are then followed by three fully connected layers, the last of which has a softmax activation and acts as output of the decoder. The convolutional kernel size was 40 and 30 for the first and second convolutional module respectively. The dropout percentage was set to $10 \%$ for both modules. The size of the first linear layer after the convolutional module was set to $50 \%$ the size of the unfolded output of the second convolutional module itself and the size of the second linear layer was set to $60 \%$ the size of the first linear layer (78 and 56 respectively) The size of the output layers is 6, i.e. the number of PoS tags used in the data.

Data of each subject was segmented in batches consisting of 32 trials per batch. One batch was held out and reserved for testing; the remaining 31 were used for hyperparameter optimization and training. Due to the small size of the per-subject datasets, the training data was used both for hyperparameter optimization (convolutional kernel size, dropout, learning rate) and training the model; the held-out test data was never used for hyperparam- 
eter optimization.



Figure 3: Convolutional module composed by one 1DCNN layer followed by dropout, batch normalization and maxpool.

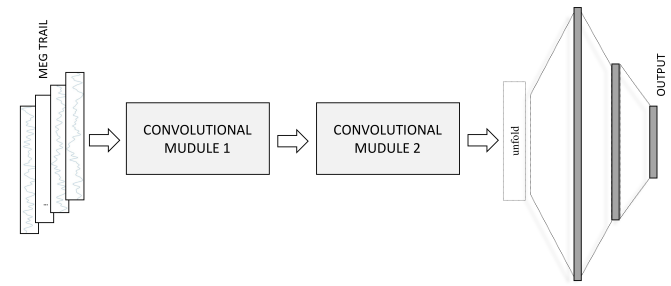

Figure 4: Complete architecture composed by 2 stacked convolutional modules followed by linear and output softamx layers.

\subsection{Training}

Training and testing is performed on each of the 15 subjects separately. The decoders are presented with a trial of MEG activity at a time $\left(T_{i}\right)$ and asked to produce one PoS tag of $w_{i}$ of $T_{i}$. Training was conducted using mini-batches of 32 trials. Training was performed on all but 1 mini-batch. The held-out batch was used for testing. Optimization is performed with the Adamax algorithm with a learning rate of 0.0002 for a maximum of 400 epochs. Early stopping was adopted as a heuristic solution to overfitting. The decoder was implemented in PyTorch.

\subsection{Results}

Figure 5 and Table 1 shows the accuracy scores of PoS decoding for all subjects. Training, validation and testing were conducted for each subject separately. The reason why each subject's data was treated as a separate dataset is that inter-subject variability is too high to allow accurate crosssubject generalization. Inter-subject variability is due to the anatomical and possibly functional differences between different people's brains. The baseline level is set at $22.74 \%$, which corresponds to the percentage of the most frequent PoS tag in the dataset (noun, see Section 3.1).

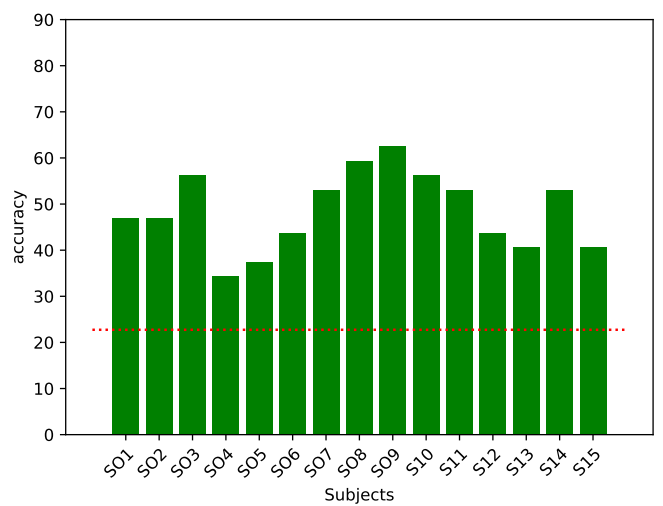

Figure 5: Test accuracies for all subjects. Horizontal dashed line indicates the baseline score of $22.74 \%$, the frequency of the most frequent PoS tag in the dataset.

$\begin{array}{ll}\text { Subject } & \text { Accuracy } \\ \text { S01 } & 46.88 \\ \text { S02 } & 46.88 \\ \text { S03 } & 56.25 \\ \text { S04 } & 34.38 \\ \text { S05 } & 37.50 \\ \text { S06 } & 43.75 \\ \text { S07 } & 53.12 \\ \text { S08 } & 59.38 \\ \text { S09 } & 62.50 \\ \text { S10 } & 56.25 \\ \text { S11 } & 53.12 \\ \text { S12 } & 43.75 \\ \text { S13 } & 40.62 \\ \text { S14 } & 53.12 \\ \text { S15 } & 40.62\end{array}$

Table 1: Test accuracies (\%) by-subject

\subsection{Error analysis}

The decoder achieves above-chance accuracy for all 15 subjects. Given the unbalanced distribution of classes in our data set we expect that misclassifications are likely to be caused by the difference between high-frequency vs. low-frequency classes. We did not use any form of weighting 
to counterbalance this bias in the output layer. Figure 6 displays a confusion matrix representing the classification of true tags (rows) into predicted tags (columns). The diagonal contains the percentage of correctly labelled trials per PoS-tag. The matrix contains the classification percentage aggregated over all 15 subjects.

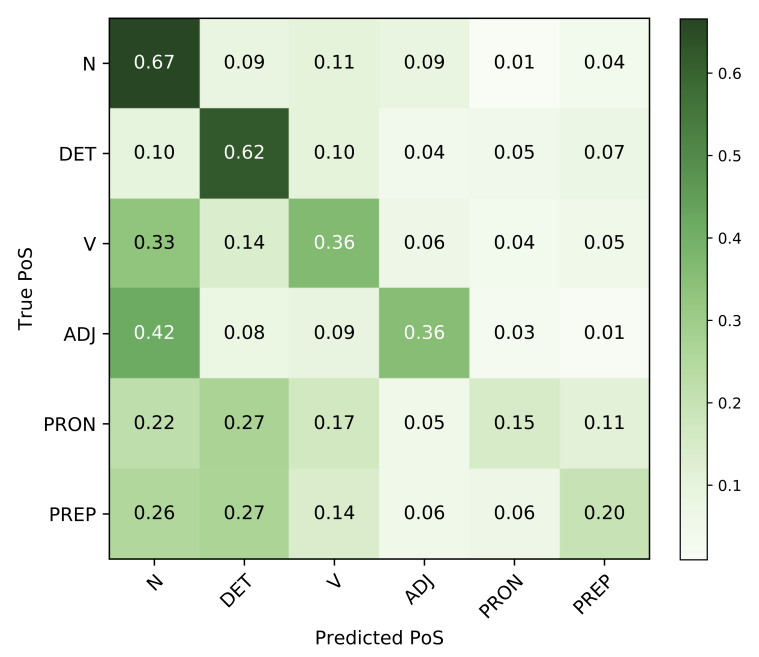

Figure 6: Confusion matrix aggregated over the 15 subjects.

The labels with higher correct classification are also the most frequent in the data set (nouns N, and determiners DET). Interestingly, content word categories (adjectives ADJ and verbs $\mathrm{V}$ ) tend to be more frequently misclassified as $\mathrm{N}$. A similar pattern applies to pronouns (PRON), which tend to be misclassified as determiners (DET).

For completeness, Figure 7 visualizes the confusion matrices for each of the 15 subjects, showing considerable variation among the subjects.

The pattern of misclassification may be due primarily to the imbalanced distribution of the PoS in the stimulus sentences. The distribution reflects intrinsic properties of natural language, where certain grammatical categories (e.g. nouns) are more frequent than others (e.g. pronouns).

Low-level perceptual characteristics of the label sets might play a role as well. Considering that classes such as $\mathrm{N}$ and ADJ, so-called content-word or open-class categories, contain words that tend to be longer than those contained in function-word or closed-class categories such as DET or PRON, it is possible that an adjective is more likely to be misclassified as a noun because of similar visual length. On the other hand, the classifier might pick up information from language processing ar- eas and therefore misclassify trials according to more similar linguistic functions, therefore ADJ tend to be confused more with $\mathrm{N}$ as compared to DET because $\mathrm{N}$ and ADJ, both content-word categories, co-occur in similar linguistic contexts, different from the contexts in which DET occurs.

\section{Conclusions}

We presented experimental results of end-to-end decoding of linguistic information from brain activity. The aim of the paper was to provide a proofof-concept of a deep-learning approach directly mapping brain data to information relative to the linguistic stimuli presented during the recording. The obtained results indicate the possibility of obtaining above-chance accuracies in classifying single MEG trials collected during sentence reading according to the PoS of their words. PoS tags were chosen as benchmark level for the presented pipeline because they represent a clean linguistic level containing purely syntactic information, and abstracting away from semantic or conceptual representations.

Error analysis suggests that both low-level visual features and higher level lexical information might be leveraged by the network in order to solve the PoS classification of the MEG trials. Error analysis indicates that content words tend to be misclassified as the most frequent content-word category, nouns, whereas function words are most frequently misclassified as determiners.

This experiment offers an example of an end-toend linguistic information decoder that relies only on the information contained in the MEG data. The above-chance scores are promising, nonetheless more work is needed to improve the overall performance of the model.

The main and most obvious limitation of the present study is represented by the relative small size of the dataset. One potential improvement could come from the introduction of contextual and sequential information relative to the input trial. Ongoing work is focused on the integration of LSTM to the present 1DCNN architecture. Moreover, In future work we plan to focus on the application to other types of linguistic information, including lexical-semantic and higher-level syntactic information. 


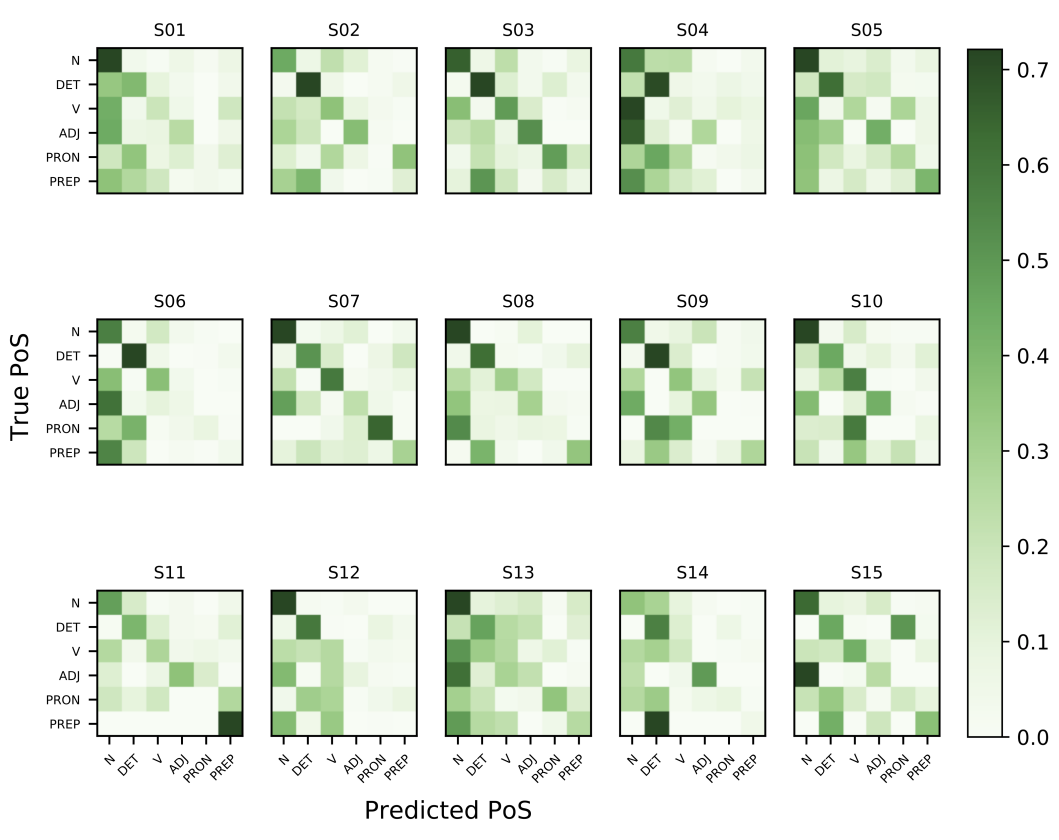

Figure 7: Confusion matrices for the 15 subjects.

\section{References}

Hassan Akbari, Bahar Khalighinejad, Jose Herrero, Ashesh Mehta, and Nima Mesgarani. 2019. Towards reconstructing intelligible speech from the human auditory cortex. Scientific Reports, 9(874).

Jeffrey R. Binder and Rutvik H. Desai. 2011. The neurobiology of semantic memory. Trends in Cognitive Sciences, 15(11):527-536.

Jonathan R. Brennan, Edward P. Stabler, Sarah E. Van Wagenen, Wen-Ming Luh, and John T. Hale. 2016. Abstract linguistic structure correlates with temporal activity during naturalistic comprehension. Brain and Language, 157-158:81-94.

Marco Catani, Derek K. Jones, and Dominic H. ffytche. 2005. Perisylvian language networks of the human brain. Annals of Neurology, 57(1):8-16.

Laurent Cohen. 2002. Language-specific tuning of visual cortex? functional properties of the visual word form area. Brain, 125(5):1054-1069.

Frank Van Eynde. 2004. Part of speech tagging en lemmatisering van het Corpus Gesproken Nederlands. Technical report, Centrum voor Computerlinguïstiek, K.U. Leuven.

Angela D. Friederici and Sarah M. E. Gierhan. 2012. The language network. Current Opinion in Neurobiology, 23:250-254.

Angela D. Friederici, Shirley-Ann Rüschemeyer, Anja Hahne, and Christian J. Fiebach. 2003. The role of left inferior frontal and superior temporal cortex in sentence comprehension: Localizing syntactic and semantic processes. Cerebral Cortex, 13(2):170.
W. Haeseryn, K. Romijn, G. Geerts, J. de Rooij, and M. van den Toorn. 1997. Algemene Nederlandse Spraakkunst. ONijhoff and Deurne: Wolters Plantyn.

Peter Hagoort. 2013. MUC (Memory, unification, control) and beyond. Front Psychology, 4:416.

James V. Haxby, M. Ida Gobbini, Maura L. Furey, Alumit Ishai, Jennifer L. Schouten, and Pietro Pietrini. 2001. Distributed and overlapping representations of faces and objects in ventral temporal cortex. Science, 293(5539):2425-2430.

Christian Herff, Dominic Heger, Adriana de Pesters, Dominic Telaar, Peter Brunner, Gerwin Schalk, and Tanja Schultz. 2015. Brain-to-text: decoding spoken phrases from phone representations in the brain. Frontiers in Neuroscience, 9:217.

Geoffrey E. Hinton, Nitish Srivastava, Alex Krizhevsky, Ilya Sutskever, and Ruslan Salakhutdinov. 2012. Improving neural networks by preventing co-adaptation of feature detectors. CoRR, abs/1207.0580.

Alexander Huth, Wendy A. de Heer, Thomas L. Griffiths, Frédéric Theunissen, and Jack Gallant. 2016. Natural speech reveals the semantic maps that tile human cerebral cortex. Nature, 532:453-458.

Sergey Ioffe and Christian Szegedy. 2015. Batch normalization: Accelerating deep network training by reducing internal covariate shift. CoRR, abs/1502.03167.

Ilya Kuzovkin, Raul Vicente, Mathilde Petton, JeanPhilippe Lachaux, Monica Baciu, Philippe Kahane, Sylvain Rheims, Juan R. Vidal, and Jaan Aru. 2018. 
Activations of deep convolutional neural networks are aligned with gamma band activity of human visual cortex. Communications Biology, 1(1):107.

Md Nasir Uddin Laskar, Luis G. Sánchez Giraldo, and Odelia Schwartz. 2018. Correspondence of deep neural networks and the brain for visual textures. CoRR, abs/1806.02888.

Tom M. Mitchell, Svetlana V. Shinkareva, Andrew Carlson, Kai-Min Chang, Vicente L. Malave, Robert A. Mason, and Marcel Adam Just. 2008. Predicting human brain activity associated with the meanings of nouns. Science, 320(5880):1191-1195.

Nelleke Oostdijk. 2000. The spoken dutch corpus. overview and first evaluation. In Proceedings of the Second International Conference on Language Resources and Evaluation (LREC-2000), Athens, Greece. European Language Resources Association (ELRA). ACL Anthology Identifier: L00-1083.

Robin Tibor Schirrmeister, Jost Tobias Springenberg, Lukas Dominique Josef Fiederer, Martin Glasstetter, Katharina Eggensperger, Michael Tangermann, Frank Hutter, Wolfram Burgard, and Tonio Ball. 2017. Deep learning with convolutional neural networks for eeg decoding and visualization. Human Brain Mapping, 38(11):5391-5420.

Jan-Mathijs Schoffelen, Robert Oostenveld, Nietzsche H. L. Lam, Julia Uddén, Annika Hultén, and Peter Hagoort. 2019. A 204-subject multimodal neuroimaging dataset to study language processing. Scientific Data, 6(17).

Zhichuan Tang, Chao Li, and Shouqian Sun. 2017. Single-trial eeg classification of motor imagery using deep convolutional neural networks. Optik, 130:11 - 18

John Thomas, Tomasz Maszczyk, Nishant Sinha, Tilmann Kluge, and Justin Dauwels. 2017. Deep learning-based classification for brain-computer interfaces. In 2017 IEEE International Conference on Systems, Man, and Cybernetics (SMC), pages 234 239.

David C. Van Essen, Matthew F. Glasser, Donna L. Dierker, John Harwell, and Timothy Coalson. 2011. Parcellations and Hemispheric Asymmetries of $\mathrm{Hu}-$ man Cerebral Cortex Analyzed on Surface-Based Atlases. Cerebral Cortex, 22(10):2241-2262. 